\title{
Precoding design for space-time codes and relay selection with a novel scheme for multiple relay systems
}

\author{
Youyan Zhang ${ }^{1,2}$ and Youming $\mathrm{Li}^{1 *}$
}

\begin{abstract}
In this paper, we consider the joint design of precoding matrices at both the source and relay nodes in a multi-relay system based on perfect and imperfect channel information, and propose the novel relay selection scheme based on log-likelihood ratio (LLR). Moreover, the symbol error rate (SER) for relay selection system based on proposed scheme is analyzed. At first, we design the combined precoding matrices based on respectively the maximum signal-to-noise ratio (SNR) with perfect channel state information (CSI) and the minimum SER of the single-relay system with imperfect CSI. Using decomposition of matrices, the joint design problems are solved with a two-step method, i.e., the first step is to determine the optimal direction of the precoding matrices, and the second step is to transform the optimization problem into two kinds of independent sub-problems which can be solved in lower complexity. Then, different from traditional relay selection based on signal-to-noise ratio, the relay node with the maximum LLR is chosen. Simulation results show the proposed selection scheme based on LLR is superior to the scheme based on SNR in the SER performance. Furthermore, the multi-relay system using the precoding matrices joint space-time coding technology can improve SER performance of the system effectively compared with only space-time coding or only precoding system.
\end{abstract}

Keywords: Precoding, Space-time block codes, Log-likelihood ratio, Relay selection

\section{Introduction}

With the rapid growth of energy demand for wireless multimedia services, communications energy consumption is growing at a remarkable rate. Therefore, how to improve the effectiveness of energy has attracted considerable attention. Cooperative relay systems can significantly increase communication system capacity and reliability. According to the number of relays, it can be divided into two categories: single-relay system and multi-relay system. Compared with the single-relay system, multi-relay system can achieve superior performance, but it also brings a sharp rise in hardware cost. One of the solutions is the relay selection strategy which selects the relay nodes to cooperate. It can improve the system performance through obtaining full diversity gain [1-6] and increasing energy efficiency.

If the channel station information (CSI) is available, the relay selection system can also adopt precoding

\footnotetext{
* Correspondence: liyouming@nbu.edu.cn

${ }^{1}$ College of Information Science and Engineering, Ningbo University, NingBo 315211, Zhejiang, China

Full list of author information is available at the end of the article
}

technology to obtain angular selectivity gain [7-11]. With all relay nodes having multiple antennas, the optimal scheme with full CSI and limited feedback scheme are proposed in [7]. Under time-varying channels, a closed-form expression for the outage probability of an amplify-and-forward (AF) relay selection system is given in [8]. Both of them select the best relay according to the signal-to-noise ratio (SNR) criterion. Recently, novel multiple relay selection schemes and beamforming for full-duplex relay networks are presented that utilize sparsity inducing optimization problems toward minimizing the mean square error (MSE) [9]. Similar to the AF protocol, decode-and-forward (DF) protocol is also one of the basic protocols in the relay system, which is investigated in the joint relay selection and precoding systems $[10,11]$. The authors of [10] study a relay selection joint with cooperative beamforming and select the best two among several relays. Meanwhile, a modified maximal ratio combining detector is proposed to achieve full diversity order. In [11], a new beamforming algorithm called channel alignment and a MAX-MAX antenna selection method 
are proposed in DF two-way relay networks. It also analyzes the bit error rate (BER) and proves that the proposed scheme can achieve full diversity order. In addition, the combination of relay selection and space-time coding has also been carried out [12, 13]. For example, in [13], a scheme coined opportunistic space-time coding to combat the outdated CSI is proposed, and the closed-form expressions of outage probability and ergodic capacity are derived.

Log-likelihood ratio (LLR) is a widely used function since it is easy to manipulate, and it has been applied in many scenarios $[14,15]$. A strategy to approximate the LLR values under higher-order constellations is proposed, which greatly reduces the complexity compared to the Max-Log-MAP soft-demapper [14]. In [15], the LLR selection is used in successive-cancelation (SC) decoding of polar codes to reduce higher implementation complexity. Some works on selection strategies based on LLR have been carried out [16-20]. In [16, 17], the selection scheme based on LLR has been proved to be better than the scheme based on SNR in symbol error rate (SER) performance for multiple-input multiple-output (MIMO) system. Due to the similarity between MIMO system and relay system, the LLR scheme is considered in the relay selection system [18-20]. The authors of [18] investigate two types of relay selection schemes, i.e., SNR-based relay selection and LLR-based relay selection. Meanwhile, the closed-form average BER for binary phase-shift keying (BPSK) signals in Nakagami-m fading channels is derived. However, it is investigated in one-way relay system. In [19], a new LLR algorithm using DF protocol in two-way networks is proposed and the BER of proposed scheme is investigated over Rayleigh fading channels.

Since the LLR criterion can bring performance improvement to the relay selection system, we combine it with precoding and space-time block codes (STBC) technology to improve the performance through obtaining spatial diversity. Here after, we call the combined technique "precoding-plus-STBC." Unlike most of the work based on LLR just from the source to the relay, we focus on the total LLR of received signal from the source to the destination. And the precoding matrices at both the source and relay nodes are designed with different CSI. Meanwhile, AF protocol is considered due to the simplicity. Due to the fact that the direct link often does not exist in the real communication environment, we consider that there is no direct link, which is different from [7]. As far as we know, the LLR scheme with precoding-plus-STBC has not been discussed in multi-relay systems.

The contributions of this paper are as follows:

- We consider the STBC combined the precoding at both the source and relay nodes, and derive the precoding matrices with perfect CSI and imperfect CSI. Different from other papers, we decompose the joint design problems into two steps to reduce the complexity: the first step is to determine the optimal direction, and the second step is to determine the optimal distribution of power in these directions.

- To improve the SER performance, we apply the log-likelihood ratio criterion to the relay selection system with precoding-plus-STBC. The superiority of the LLR is explored, and the criterion of relay selection based on LLR is derived. Compared with the traditional selection scheme based on SNR, the scheme based on LLR obtains the better SER performance.

- The SER of relay selection system based on LLR is derived. It is proved that the SER is minimized compared with the SNR scheme, which is consistent with the following simulation results.

The rest of this paper is organized as follows. In Section 2, we introduce the wireless system model. In Section 3, we design the precoding matrices with perfect CSI and imperfect CSI. Section 4 proposes the relay selection scheme based on LLR and analyzes the SER performance of the relay selection system. Experimental is presented in Section 5. Results and discussion are shown in Section 6, and Section 7 draws the conclusions.

\section{System model}

We consider a two-hop multiple-input multiple-output (MIMO) cooperative system with relay selection as shown in Fig. 1, which is composed of a single source node, a single destination node, and a set of I relay nodes. Each node is equipped with $N_{\mathrm{S}}, N_{\mathrm{D}}$, and $N_{\mathrm{R}, i}(i=1,2, \ldots, \mathrm{I})$ antennas respectively. The STBC technique is used at the source and relay nodes to achieve diversity gain.

The signal transmission can be divided into two phases. In the first phase, the source node sends the data to all relays. In the second phase, the selected relay with the best log-likelihood ratio sends the symbols to the destination node. At first, the transmitted signals $\mathbf{s}=\left[s_{1}, \cdots, s_{L}\right]$ are mapped to a STBC matrix $\mathbf{G}_{1}(s) \in C^{N_{\mathrm{S}} \times T}$, and the source employs the codeword $\mathbf{G}_{1}(s)$, where $N_{\mathrm{S}}$ is the space dimension and $T$ is the time dimension. Without loss of generality, we assume the average energy of the signal constellation is equal to 1 , i.e., $E\left[\left|s_{l}\right|^{2}\right]=1, l \in\{1, \cdots, L\}$. The received signal at the $i$ th relay is given by

$$
\mathbf{Y}_{\mathrm{R}, i}=\mathbf{H}_{1, i} \mathbf{V}_{1} \mathbf{G}_{1}(s)+\mathbf{N}_{1, i}
$$

where $\mathbf{H}_{1, i} \in C^{N_{\mathrm{R}, i} \times N_{\mathrm{S}}}$ is the MIMO channel between the source and the $i$ th relay, $\mathbf{V}_{1} \in C^{N_{S} \times N_{S}}$ is the source precoding matrix, and $\mathbf{N}_{1, i}$ is a sample matrix of the additive white Gaussian noise (AWGN) with mean zero and variance $\tilde{\sigma}_{1}^{2}$. 


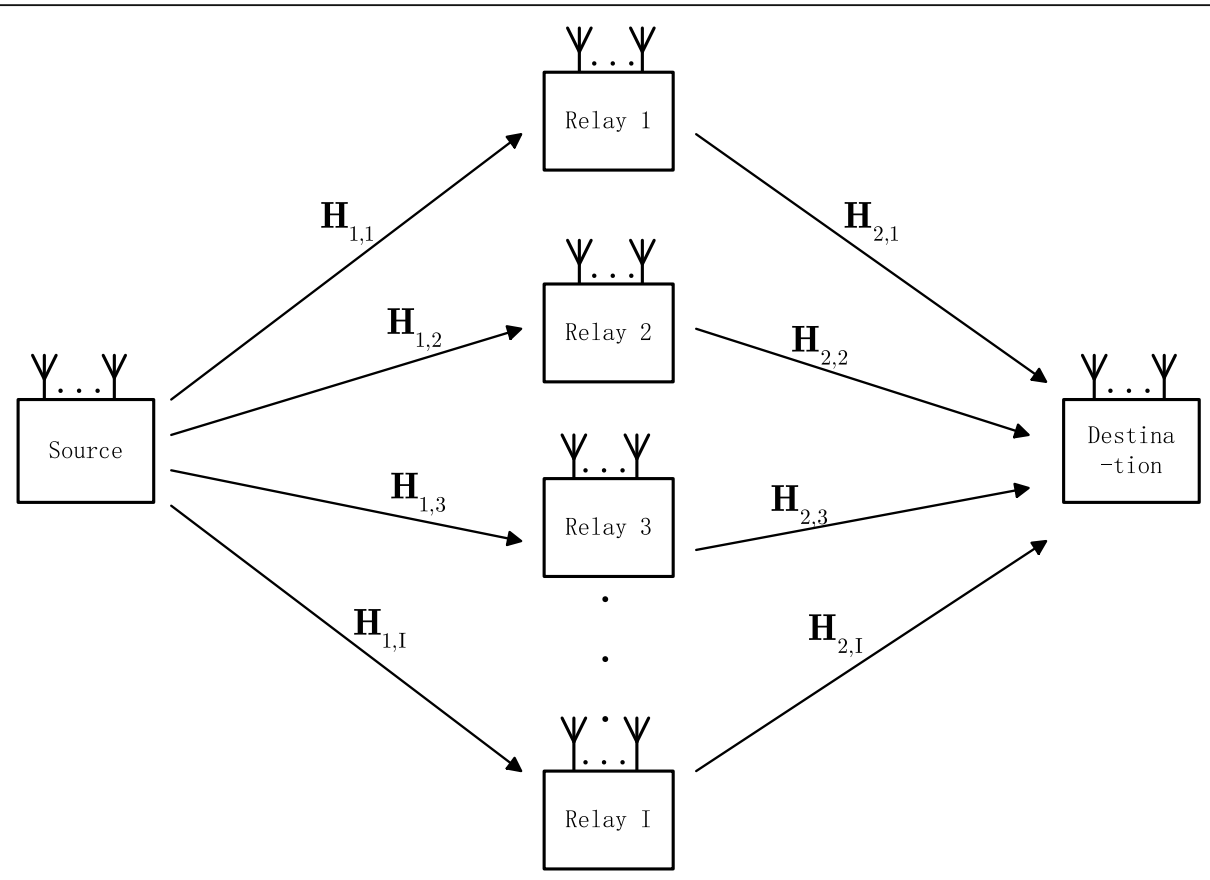

Fig. 1 System model

We transform a MIMO fading channel into $L$ parallel SISO channels using the orthogonality of the STBC. Hence, the signal at the $i$ th relay node on the $l$ th channel is denoted by

$$
y_{l}^{\mathrm{R}, i}=\left\|\mathbf{H}_{1, i} \mathbf{V}_{1}\right\| s_{l}+n_{l}^{\mathrm{R}, i}
$$

where $n_{l}^{\mathrm{R}, i} \sim \mathrm{CN}\left(0, \sigma_{1}^{2}=\tilde{\sigma}_{1}^{2} / c\right), c$ is the code constant [21]. For the AF protocol, the received signal at the $i$ th relay can be normalized by a factor of energy

$$
t_{l}=y_{l}^{\mathrm{R}, i} / E^{1 / 2}\left\{\left|y_{l}^{\mathrm{R}, i}\right|^{2}\right\}
$$

In the second phase, the selected relay encodes $t_{l}$ to a STBC matrix $\mathbf{G}_{2}(t)$, then multiplies the precoding matrix, and forwards the signal to the destination. The final signal is given by

$$
\mathbf{Y}_{D}=\mathbf{H}_{2, i} \mathbf{V}_{2, i} \mathbf{G}_{2}(t)+\mathbf{N}_{2, i}
$$

where $\mathbf{H}_{2, i} \in C^{N_{\mathrm{D}} \times N_{\mathrm{R}, i}}$ is the channel matrix of the relay to destination, $\mathbf{G}_{2}(t) \in C^{N_{\mathrm{R}, i} \times T}, \mathbf{V}_{2, i} \in C^{N_{\mathrm{R}, i} \times N_{\mathrm{R}, i}}$ is the $i$ th relay precoding matrix, and $\mathbf{N}_{2,} i$ is a sample matrix of AWGN with mean zero and variance $\tilde{\sigma}_{2}^{2}$. Similar to (1), the signal received by the destination on the $l$ th channel can be written as

$$
y_{l}^{\mathrm{D}, i}=\left\|\mathbf{H}_{2, i} \mathbf{V}_{2, i}\right\| t_{l}+n_{l}^{\mathrm{D}, i}
$$

where $n_{l}^{D, i} \sim \mathrm{CN}\left(0, \sigma_{2}^{2}=\tilde{\sigma}_{2}^{2} / c\right)$. Substituting (2) and (3) into (5) gives

$$
y_{l}^{\mathrm{D}, i}=\underbrace{\frac{\left\|\mathbf{H}_{1, i} \mathbf{V}_{1}\right\|\left\|\mathbf{H}_{2, i} \mathbf{V}_{2, i}\right\| s_{l}}{\sqrt{\left\|\mathbf{H}_{1, i} \mathbf{V}_{1}\right\|^{2}+\sigma_{1}^{2}}}}_{\text {Signal }}+\underbrace{\frac{\left\|\mathbf{H}_{2, i} \mathbf{V}_{2, i}\right\| n_{l}^{\mathrm{R}, i}}{\sqrt{\left\|\mathbf{H}_{1, i} \mathbf{V}_{1}\right\|^{2}+\sigma_{1}^{2}}}+n_{l}^{\mathrm{D}, i}}_{\text {Noise }}
$$

By employing the maximum ratio combining (MRC) criterion at the destination, the total output SNR has the following form:

$$
\begin{aligned}
\gamma_{\mathrm{SNR}} & =\frac{\left\|\mathbf{H}_{1, i} \mathbf{V}_{1}\right\|^{2}\left\|\mathbf{H}_{2, i} \mathbf{V}_{2, i}\right\|^{2}}{\left\|\mathbf{H}_{1, i} \mathbf{V}_{1}\right\|^{2} \sigma_{2}^{2}+\left\|\mathbf{H}_{2, i} \mathbf{V}_{2, i}\right\|^{2} \sigma_{1}^{2}+\sigma_{1}^{2} \sigma_{2}^{2}} \\
& =\frac{\gamma_{1} \gamma_{2}}{1+\gamma_{1}+\gamma_{2}}
\end{aligned}
$$

where $\gamma_{1}=\left\|\mathbf{H}_{1, i} \mathbf{V}_{1}\right\|^{2} / \sigma_{1}^{2}, \quad \gamma_{2}=\left\|\mathbf{H}_{2, i} \mathbf{V}_{2, i}\right\|^{2} / \sigma_{2}^{2}$.

Similar result is also derived in $[22,23]$, but only single relay in the systems is discussed.

\section{Precoding matrices with different CSI}

The work on precoding-plus-STBC design has been done in [21], which aim at the precoding matrix in MIMO systems. However, in this paper, we extend it to the relay system. Compared with the MIMO system, the precoding problem to be solved is more challenging. That is different from [21]. The precoding matrices are used both at the source and relay nodes, and the design is a joint problem. Therefore, we decompose the joint function by analyzing the properties of the total received 
SNR and the SER performance of the relay system, and reduce the complexity of the problem by two steps.

\subsection{Precoding matrices with perfect CSI}

In this section, our purpose is to find the precoding matrices $\mathbf{V}_{1}$ in the source and $\mathbf{V}_{2, i}$ in the $i$ th relay node which can maximize the total SNR $\gamma_{\mathrm{SNR}}$. Assuming there are high-speed feedback links between transmitters and receivers, the transmitters can get perfect CSI, and the system can get the better SER by increasing the received SNR. For simplicity, $\mathrm{X}_{n, i}$ in the previous section is rewritten as $\mathrm{X}_{n}$, in this case $\mathbf{V}_{2}=\mathbf{V}_{2, i}$. Therefore, the optimization problem can be formulated as

$$
\begin{aligned}
& \max _{\mathbf{V}_{1}, \mathbf{V}_{2}} \gamma_{\mathrm{SNR}} \\
& \text { s.t. } \quad L \cdot c \cdot \operatorname{tr}\left\{\mathbf{V}_{k}^{H} \mathbf{V}_{k}\right\}=P_{k} \quad k=1,2
\end{aligned}
$$

where $L$ is the number of parallel channels, $P_{1}$ and $P_{2}$ are respectively the transmit power constraints at the source and the selected relay nodes, and $\operatorname{tr}\{\cdot\}$ is the trace of matrix. To solve the problem (8) efficiently, the expression of $\gamma_{\mathrm{SNR}}$ should be analyzed from Proposition 1, which is based on the following Lemma 1 .

Lemma 1 For two non-negative variables $x$ and $y$ with $x \in(0, A], y \in(0, B]$, the function $f(x, y)=x y /(1+x+y)$ is a monotonically increasing function.

Proof First, take partial derivative of $f(x, y)$ with respect to $x$ and $y$, we have $f_{x}^{\prime}(x, y)=\frac{y^{2}+y}{(1+x+y)^{2}}>0$ and $f_{y}^{\prime}(x, y)$ $=\frac{x^{2}+x}{(1+x+y)^{2}}>0$. Then, we need to have the directional derivative, which means to prove the function is monotonically increased in any direction. Using $y=k x, k>0$, take partial derivative of $f(x, y)$ with respect to $x$, we get $f_{x}^{\prime}(x$, $k x)=\frac{2 k x+k x^{2}+k^{2} x^{2}}{(1+x+k x)^{2}}>0$. With partial derivative and directional derivative, the function $f(x, y)$ is proved to be increasing monotonically.

This completes the proof of Lemma 1 .

Proposition 1 For non-negative independent random variables $\gamma_{1}$ and $\gamma_{2}$ as defined in Eq. (7), the total SNR $\gamma_{\mathrm{SNR}}$ achieves the maximum when both $\gamma_{1}$ and $\gamma_{2}$ are maximum.

Proof Using the results from Lemma 1, we can draw a conclusion that the function $\gamma_{\mathrm{SNR}}$ is a monotonically increasing function. Since $\gamma_{1}$ and $\gamma_{2}$ are non-negative independent random variables and have the maximum values, the maximum of $\gamma_{\mathrm{SNR}}$ is achieved when $\gamma_{1}=\max$ $\gamma_{1}$ and $\gamma_{2}=\max \gamma_{2}$. Therefore, the optimization problem $\max \gamma_{\mathrm{SNR}}$ can be divided into two sub-problems $\max \gamma_{1}$ and $\max \gamma_{2}$.

This completes the proof of Proposition 1.

Based on Proposition 1, the problem (8) can be divided into two simplified sub-problemsmax $\gamma_{1}$ and $\max \gamma_{2}$ as

$$
\begin{aligned}
& \max _{\mathbf{V}_{k}}\left\|\mathbf{H}_{k} \mathbf{V}_{k}\right\|^{2} \\
& \text { s.t. } \quad L \cdot c \cdot \operatorname{tr}\left\{\mathbf{V}_{k}^{H} \mathbf{V}_{k}\right\}=P_{k} \quad k=1,2
\end{aligned}
$$

This optimization problem can be solved by a two-step method: the first step is to determine the directions of the two precoding matrices, and the second step is to allocate the power in each direction.

Without loss of generality, we assume the precoding matrices can be decomposed as follows: $\mathbf{V}_{k}=\mathbf{U}_{\mathbf{V}_{k}} \mathbf{D}_{\mathbf{V}_{k}} \mathbf{U}_{\mathbf{V}_{k}}^{H}$ $k=1,2$, which is the eigen-decomposition of matrix $\mathbf{V}_{k}$. Let $\mathbf{H}_{k}{ }^{H} \mathbf{H}_{k}=\mathbf{U}_{\mathbf{H}_{k}} \mathbf{D}_{\mathbf{H}_{k}} \mathbf{U}_{\mathbf{H}_{k}}^{H}$ be the eigen-decomposition of $\mathbf{H}_{k}{ }^{H} \mathbf{H}_{k}$. From [24], it is seen that $\left\|\mathbf{H}_{k} \mathbf{V}_{k}\right\|^{2}$ is maximized when $\mathbf{U}_{\mathbf{V}_{k}}=\mathbf{U}_{\mathbf{H}_{k}}$. Therefore, the optimization direction of the precoding matrices is along with the corresponding eigenvector of channel matrices. Next, the power allocation is considered in each direction.

Using the eigen-decompositions of $\mathbf{V}_{k}$ and $\mathbf{H}_{k}{ }^{H} \mathbf{H}_{k}$, expression (9a) can be formulated as

$$
\begin{aligned}
\left\|\mathbf{H}_{k} \mathbf{V}_{k}\right\|^{2}=\operatorname{tr}\left(\mathbf{V}_{k} \mathbf{V}_{k}^{H} \mathbf{H}_{k}^{H} \mathbf{H}_{k}\right) \\
\quad=\operatorname{tr}\left(\mathbf{U}_{\mathbf{V}_{k}} \mathbf{D}_{\mathbf{V}_{k}} \mathbf{U}_{\mathbf{V}_{k}}^{H}\left(\mathbf{U}_{\mathbf{V}_{k}} \mathbf{D}_{\mathbf{V}_{k}} \mathbf{U}_{\mathbf{V}_{k}}^{H}\right)^{H} \mathbf{U}_{\mathbf{H}_{k}} \mathbf{D}_{\mathbf{H}_{k}} \mathbf{U}_{\mathbf{H}_{k}}^{H}\right) \\
=\operatorname{tr}\left(\mathbf{U}_{\mathbf{H}_{k}} \mathbf{D}_{\mathbf{V}_{k}}^{2} \mathbf{D}_{\mathbf{H}_{k}} \mathbf{U}_{\mathbf{H}_{k}}^{H}\right)=\operatorname{tr}\left(\mathbf{D}_{\mathbf{V}_{k}}^{2} \mathbf{D}_{\mathbf{H}_{k}}\right)
\end{aligned}
$$

Therefore, the optimization problem (9) can be converted to linear programming problem.

$$
\begin{aligned}
& \mathbf{D}_{\mathbf{V}_{k, \text { opt }}}=\arg \max \operatorname{tr}\left(\mathbf{D}_{\mathbf{V}_{k}}^{2} \mathbf{D}_{\mathbf{H}_{k}}\right) \\
& \text { s.t. } \quad L \cdot c \cdot \operatorname{tr}\left\{\mathbf{V}_{k}^{H} \mathbf{V}_{k}\right\}=P_{k} \quad k=1,2
\end{aligned}
$$

This linear programming problem can be solved by some common software easily, such as Excel, Lindo, and Matlab. The solution shows that the optimal power allocation scheme is to load all the power on the direction corresponding to the maximum eigenvalue of the MIMO channel matrix, while the power in other directions is zero.

\subsection{Precoding matrices with imperfect CSI}

In this section, we want to find the precoding matrices with the statistical CSI. Assuming a flat block-fading correlated Rayleigh fading channel model, the autocorrelation coefficients of the channel with Kronecker product is given by [21, 25] $\mathbf{R}_{\mathbf{H}_{k}}=\mathbf{R}_{\mathrm{tk}}^{T} \otimes \mathbf{R}_{\mathrm{rk}} \quad k=1,2$, where $\mathbf{R}_{\mathrm{t} 1}$ and $\mathbf{R}_{\mathrm{r} 1}$ are the transmit and receive correlation matrices for the source-to-relay link. Similarly, $\mathbf{R}_{\mathrm{t} 2}$ and $\mathbf{R}_{\mathrm{r} 2}$ are the transmit and receive correlation matrices for the relay-to-destination link.

Since both the channels $\mathbf{H}_{1, i}$ and $\mathbf{H}_{2, i}$ are unknown in (4), the precoding matrices are designed with a different 
objective function. Considering the complexity of the symbol error rate of the relay selection system with precoding-plus-STBC, we design the matrices which minimize the SER of the single-relay system. That is to say, first, the precoding matrix of each relay is obtained according to the objective function, and then, the relay selection is carried out. The SER expression of the single relay with precoding-plus-STBC is given by [23]

$$
\begin{aligned}
\mathrm{P}_{s}(r) \approx a \int_{0}^{b \pi}\left[\sum_{k=1}^{2} \operatorname{det}^{-1}\left(\mathbf{I}_{N_{\mathrm{t} k} N_{\mathrm{r} k}}+\mathbf{A}_{k} g / \sigma_{k}^{2} \sin ^{2} \theta\right)\right. \\
\left.-\prod_{k=1}^{2} \operatorname{det}^{-1}\left(\mathbf{I}_{N_{\mathrm{t} k} N_{\mathrm{r} k}}+\mathbf{A}_{k} g / \sigma_{k}^{2} \sin ^{2} \theta\right)\right] d \theta
\end{aligned}
$$

where $N_{\mathrm{t} 1}$ and $N_{\mathrm{r} 1}$ denote the number of transmit and receive antennas corresponding to the channel $\mathbf{H}_{1, i}$ respectively. While $N_{\mathrm{t} 2}$ and $N_{\mathrm{r} 2}$ denote the number of transmit and receive antennas corresponding to the channel $\mathbf{H}_{2, i}$ respectively. The parameters $a, b$ and $g$ are determined by the specific modulation scheme, such as for MPSK, $a=1 / \pi, b=(M-1) / M$, and $g=\sin ^{2}(\pi / M)$. And $\mathbf{A}_{i}$ is defined as $\mathbf{A}_{i}=\mathbf{R}_{\mathbf{H}_{i}}^{1 / 2}\left(\mathbf{V}_{i}{ }^{*} \mathbf{V}_{i}^{T} \otimes \mathbf{I}_{N_{r i}}\right) \mathbf{R}_{\mathbf{H}_{i}}^{1 / 2}$. Then, this optimization problem can be formulated as

$$
\begin{aligned}
& \min _{\mathbf{V}_{1}, \mathbf{V}_{2}} \mathrm{P}_{s}(r) \\
& \text { s.t. } \quad L \cdot c \cdot \operatorname{tr}\left\{\mathbf{V}_{k}^{H} \mathbf{V}_{k}\right\}=P_{k} \quad k=1,2
\end{aligned}
$$

The definitions of $L, c$, and $P_{k}$ are the same in (8b).

Inspired by [23], we get a solution to solve this problem: the first step is to determine the directions, and the second step is to allocate the power in each direction. When the optimal direction of precoding matrices is along the eigenvectors of the transmit correlation matrices, the minimum SER is achieved, i.e., the precoding matrix can be decomposed as follows, $\mathbf{V}_{k}=\mathbf{U}_{\mathbf{R}_{t k}} \mathbf{W}_{\mathbf{V}_{k}}$, where $\mathbf{U}_{\mathbf{R}_{t k}}$ is the orthogonal eigenvector matrix of $\mathbf{R}_{t k}$ and $\mathbf{W}_{\mathbf{V}_{k}}$ is a diagonal matrix which determines the power allocation.

The transmit power allocation problem (13) can be divided into the following two sub-problems

$$
\max _{\mathbf{V}_{k}} \sum_{n=1}^{N_{\mathrm{rk}}} \sum_{m=1}^{N_{\mathrm{t} k}} \ln \left(1+\left(g / 4 \sigma_{k}^{2}\right) \cdot \lambda_{n}\left(\mathbf{R}_{\mathrm{r} k}\right) \lambda_{m}\left(\mathbf{R}_{\mathrm{t} k}\right) \lambda_{m}\left(\mathbf{P}_{\mathbf{V}_{k}}\right)\right)
$$

$$
\text { s.t. } \quad L \cdot c \cdot \operatorname{tr}\left\{\mathbf{V}_{k}^{H} \mathbf{V}_{k}\right\}=P_{k} \quad k=1,2
$$

where $\lambda(\mathbf{M})$ is the generalized eigenvalue of the matrix $\mathbf{M}$ and $\mathbf{P}_{\mathbf{V}_{k}}=\mathbf{V}_{k} \mathbf{V}_{k}^{H}$. For problem (14a-b), it is similar in $[21,26]$, so we can get the solution method easily.

\section{Relay selection scheme and analysis of the SER} 4.1 Relay selection scheme based on log-likelihood ratio In this section, the relay selection scheme based on log-likelihood ratio is proposed. First, we derive the scheme in multiple-input single-output (MISO) channels. From that, we derive the scheme in relay networks. It can be described as follows: the first step is to calculate the magnitude of the LLR for each relay in the destination, and the second step is to arrange the values of the LLR in descending order and select the largest LLR value to send the signal.

We define the symbol log-likelihood ratio $\operatorname{LLR}_{j} \tilde{k}^{(\tilde{m})}$ from the $\tilde{k}$ th transmit antenna to receive antenna $j$ as

$$
\operatorname{LLR}_{j \tilde{k}}(\tilde{m})=\ln \frac{\operatorname{Pr}\left(a=\hat{a}_{\tilde{j}} \mid y_{j \tilde{k}}, h_{j \tilde{k}}\right)}{\operatorname{Pr}\left(a=s_{\tilde{m}} \mid y_{j \tilde{k}}, h_{j \tilde{k}}\right)}
$$

where $\hat{a}_{j} \tilde{k}$ is the MAP decision of transmitter signal from the transmit antenna $\tilde{k}$ to the $j$ th receive antenna, i.e.,

$$
\hat{a}_{j \tilde{k}}=\arg \max _{s_{\tilde{m}} \in\left\{s_{1}, s_{2}, \cdots, s_{M}\right\}} \operatorname{Pr}\left(a=s_{\tilde{m}} \mid y_{j \tilde{k}}, h_{j \tilde{k}}\right)
$$

where $\operatorname{Pr}\left(a=s_{\tilde{m}} \mid y_{j} \tilde{k}_{k}, h_{j}\right)$ is the posteriori probability for $a=s_{\tilde{m}}$ with the receive signal $y_{j{ }_{j}}$ and the channel $h_{j} \tilde{k} \cdot\left\{s_{1}, s_{2}, \cdots s_{M}\right\}$ is the collection of modulated M-ary signals. After some manipulations, the conditional probability of symbol error for selecting antenna $\tilde{k}$, given $y_{j} \tilde{k}$ and $h_{j} \tilde{k}$, can be expressed as [27]

$$
\operatorname{Pr}\left(a \neq \hat{a}_{j \tilde{k}} \mid y_{j \tilde{k}}, h_{j \tilde{k}}\right)=1-\frac{1}{\sum_{\tilde{m}=1}^{M} e^{-L^{L L} \tilde{k}_{j}(\tilde{m})}}
$$

Therefore, the LLR-based scheme that minimizes the probability of symbol error is to select the transmit antenna that minimizes $\sum_{\tilde{m}=1}^{M} e^{-\mathrm{LLR}} \tilde{k}^{(\tilde{m})}$. According to [17], formula (15) can be transformed to

$$
\operatorname{LLR}_{j \tilde{k}}(\tilde{m})=\frac{\left(\left\|y_{j \tilde{k}}-h_{j \tilde{k}} S_{\tilde{m}}\right\|^{2}-\left\|y_{j \tilde{k}}-h_{j \tilde{k}} \hat{a}_{j \tilde{k}}\right\|^{2}\right)}{N_{0}}
$$

Then, the expression can be written as

$$
\sum_{\tilde{m}=1}^{M} e^{-\operatorname{LLR}_{j \bar{k}}(\tilde{m})}=e^{\left\|y_{j \tilde{k}}-h_{j \tilde{k}} \hat{a}_{j \bar{k}}\right\|^{2} / N_{0}} \cdot \sum_{\tilde{m}=1}^{M} e^{-\left\|y_{j \tilde{k}}-h_{j \tilde{k}} s_{\tilde{m}}\right\|^{2} / N_{0}}
$$

where $N_{0}$ is the variance of additive complex Gaussian white noise on the receive antenna. When the signals become BPSK signals, the LLR reduces to 


$$
\operatorname{LLR}_{j \tilde{k}}(\tilde{m})=\left\{\begin{array}{cc}
\frac{4 \sqrt{E_{s}}}{N_{0}}\left|y_{j \tilde{k}} h_{j \tilde{k}}\right| & s_{\tilde{m}} \neq \hat{a}_{j \tilde{k}} \\
0 & s_{\tilde{m}}=\hat{a}_{j \tilde{k}}
\end{array}\right.
$$

The selected transmit antenna is given as

$$
i_{\text {antenna }}=\arg \max \left|y_{j \tilde{k}} h_{j \tilde{k}}\right|
$$

For the relay system above, let $y_{j \tilde{k}}=y_{l}^{\mathrm{D}, i}, h_{j} \tilde{k}$ $=\frac{\left\|\mathbf{H}_{1, i} \mathbf{V}_{1}\right\|\left\|\mathbf{H}_{2, i} \mathbf{V}_{2, i}\right\|}{\sqrt{\left\|\mathbf{H}_{1, i} \mathbf{V}_{1}\right\|^{2}+\sigma_{1}^{2}}}$, and the relay selection criterion based on LLR is achieved. For BPSK signals, the selected relay is chosen according to

$$
i_{\text {relay }}=\arg \max \left|\frac{\left\|\mathbf{H}_{1, i} \mathbf{V}_{1}\right\|\left\|\mathbf{H}_{2, i} \mathbf{V}_{2, i}\right\|}{\sqrt{\left\|\mathbf{H}_{1, i} \mathbf{V}_{1}\right\|^{2}+\sigma_{1}^{2}}} \cdot y_{l}^{\mathrm{D}, i}\right|
$$

\subsection{Average SER analysis of the relay selection system}

Below, we give the analysis to the average SER of the relay selection with LLR scheme and prove that the proposed scheme can minimize the SER. Let $\mathbf{s}$ be the transmitted symbol, and $\hat{\mathbf{s}}$ is the final decision signal. The probability of symbol error rate is given by [17].

$$
\mathrm{P}_{\text {error }}=\int_{\mathbf{y}, \mathbf{h}} \operatorname{Pr}(\mathbf{s} \neq \hat{\mathbf{s}} \mid \mathbf{y}, \mathbf{h}) f_{\mathbf{y}, \mathbf{h}}(\mathbf{y}, \mathbf{h}) d \mathbf{y} d \mathbf{h}
$$

where $\quad \mathbf{y}=\left(y_{l}^{\mathrm{D}, 1}, y_{l}^{\mathrm{D}, 2}, \ldots, y_{l}^{\mathrm{D}, I}\right), \mathbf{h}=\left(h_{1}, h_{2}, \ldots, h_{I}\right), h_{i}$ $=\frac{\left\|\mathbf{H}_{1, i} \mathbf{V}_{1}\right\|\left\|\mathbf{H}_{2, i} \mathbf{V}_{2, i}\right\|}{\sqrt{\left\|\mathbf{H}_{1, i} \mathbf{V}_{1}\right\|^{2}+\sigma_{1}^{2}}}$, and $f_{\mathbf{y}, \mathbf{h}}(\mathbf{y}, \mathbf{h})$ is the joint pdf of $(\mathbf{y}, \mathbf{h})$.

Then, from (23), it can be shown that the probability of symbol error $\mathrm{P}_{\text {error }}$ is minimized by minimizing $\operatorname{Pr}(\mathbf{s} \neq \hat{\mathbf{s}} \mid$ $\mathbf{y}, \mathbf{h})$ for all $\mathbf{y}$ and $\mathbf{h}$.

$$
\begin{aligned}
\operatorname{Pr}(\mathbf{s} \neq \hat{\mathbf{s}} \mid \mathbf{y}, \mathbf{h})=\sum_{i=1}^{I} \operatorname{Pr}\left(\mathbf{s} \neq \hat{\mathbf{s}}_{i} \mid \text { ith relay selected }, \mathbf{y}, \mathbf{h}\right) \\
. \operatorname{Pr}(i \text { th relay selected } \mid \mathbf{y}, \mathbf{h}) \\
=\operatorname{Pr}\left(\mathbf{s} \neq \hat{\mathbf{s}}_{1} \mid y_{l}^{\mathrm{D}, 1}, h_{1}\right) \cdot \operatorname{Pr}(1 \text { th relay selected } \mid \mathbf{y}, \mathbf{h})+ \\
\quad \ldots \operatorname{Pr}\left(\mathbf{s} \neq \hat{\mathbf{s}}_{I} \mid y_{l}^{\mathrm{D}, I}, h_{I}\right) \cdot \operatorname{Pr}(\text { Ith relay selected } \mid \mathbf{y}, \mathbf{h}) \\
\geq \min \operatorname{Pr}\left(\mathbf{s} \neq \hat{\mathbf{s}}_{i} \mid y_{l}^{\mathrm{D}, i}, h_{i}\right) \cdot \sum_{i=1}^{I} \operatorname{Pr}(i \text { th relay selected } \mid \mathbf{y}, \mathbf{h})
\end{aligned}
$$

Notice $\sum_{i=1}^{I} \operatorname{Pr}(i$ th relay selected $\mid \mathbf{y}, \mathbf{h})=1$, and then, the equality in (24) is achieved when the relay which can minimize the $\operatorname{Pr}\left(\mathbf{s} \neq \hat{\mathbf{s}}_{i} \mid y_{l}^{\mathrm{D}, i}, h_{i}\right)$ is selected. From (16) by letting $\hat{a}_{j} \tilde{j}_{k}=\hat{\mathbf{s}}_{i}, y_{j \tilde{k}}=y_{l}^{\mathrm{D}, i}, h_{{ }_{j} k}=h_{i}$, the MAP decision rule maximizing $\operatorname{Pr}\left(\mathbf{s}=\hat{\mathbf{s}}_{i} \mid y_{l}^{\mathrm{D}, i}, h_{i}\right)$, can be obtained in the relay selection system. So the probability of symbol error $\mathrm{P}_{\text {error }}$ is minimized by selecting the relay minimizing $\operatorname{Pr}(\mathbf{s}$ $\left.\neq \hat{\mathbf{s}}_{i} \mid y_{l}^{\mathrm{D}, i}, h_{i}\right)$.
From the selection criteria, the best relay of the received signal and channel coefficient will be selected, which directly affects the SER of the system. Therefore, it can be seen obviously that compared with SNR-based relay selection, LLR-based relay selection is superior in the SER performance of the system. In addition, we will compare our proposed criteria with only precoding system and only STBC system. If only precoding is employed, the system cannot obtain the gain of diversity from STBC, and if only STBC is employed, the system cannot allocate the transmit power optimally. Therefore, the optimal error performance of relay system cannot be obtained, and then, these will be proved in the following simulation.

\section{Experimental}

The simulation is carried out in MATLAB. We consider a relay selection system with $N_{S}=N_{R, i}=N_{D}=2$. The simulation parameters are as follows: the performance of the proposed scheme is evaluated for the BPSK modulation, the Alamouti coding is employed by the source and the relay nodes, the correlation coefficient between the antennas $k$ and $j$ can be expressed as $\rho_{k j}$, and the correlation matrix be given by $\mathbf{R}=\left(\rho_{k j}^{|k-j|}\right)$. We compare the proposed method based on LLR with the conventional relay selection based on SNR with different correlation fading coefficient and the numbers of relays. Moreover, the performance of the proposed scheme is compared with the only precoding system and the only STBC system.

\section{Results and discussion}

We now examine two scenarios: perfect CSI and imperfect CSI.

\subsection{Scenario 1}

Next, the five figures show the SER versus SNR performance with perfect channels.

Figures 2 and 3 compare the proposed method based on LLR with the conventional relay selection based on SNR which is also used in [7]. But SNR scheme has not been discussed in the precoding-plus-STBC system. Figure 2 shows the SER versus SNR for different numbers of relays in correlated channels when $\rho_{k j}=0.8$. Note that the scheme LLR-based provides significant performance improvement compare to the scheme SNR-based. As the number of relay nodes I increases, the performance of this cooperative system also increases. Furthermore, when $\rho_{k j}=0.8$, the performance of the relay selection LLR-based system with $I=3$ is superior to that SNR-based with $I=4$. When the number of relays is 3 , the performances of the relay system with different correlation fading coefficient are compared in Fig. 3. The figure shows that the performance improvement decreases while the correlation 


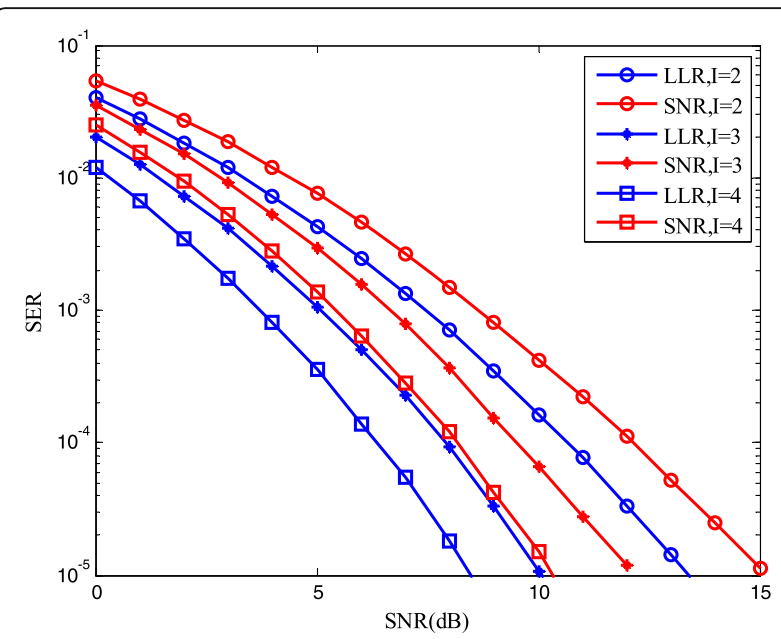

Fig. 2 Comparison between the performance of LLR-based and SNR-based relay selection in correlated channels with $\rho_{k j}=0.8$

fading coefficient increases. And the gap is the biggest when the channels are independent.

In Fig. 4 , we respectively set $\rho_{k j}=0.3,0.8,0.95$ and $I=2$ then compare the SER performance of the precoding-plus-STBC (PPS) system and only precoding (OP) system. The combination of precoding design and relay selection based on SNR has been used in [7], but the combination with relay selection based on LLR has not been discussed. For convenience of comparison, we select the best relay with LLR criterion in the two systems mentioned above. And the direct link is not considered, which is different from [7] because the direct link often does not exist in actual communication environment. It is observed from the results that as the correlation becomes stronger the performance gain also increases. So the proposed

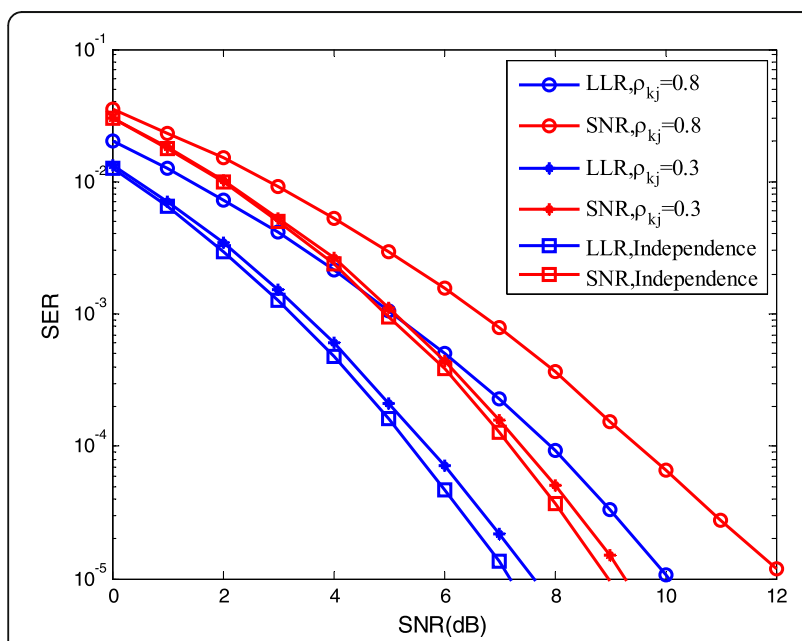

Fig. 3 SER performance of the relay selection system with different correlation fading coefficient

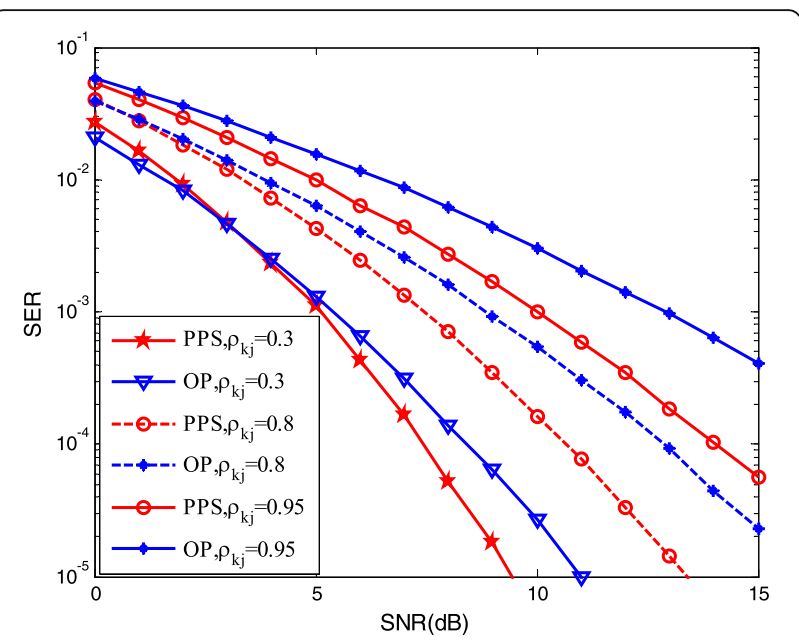

Fig. 4 Comparison between the performance of the precoding-plusSTBC and only precoding relay system with perfect CSI

precoding-plus-STBC technology can improve the SER performance effectively, especially in a highly correlated environment.

Simulation results in Fig. 5 illustrate the SER performance of the precoding-plus-STBC system and only STBC (OS) system when the number of relays $I$ is 2 . The only STBC system is discussed in [28]. The difference is that we extend the single relay in [28] to multiple relays and adopt the relay selection based on LLR. We investigate the case of $\rho_{k j}=0.95,0.3$ and independence. Simulation results show that the performance of the precoding-plus-STBC system is superior to that of the only STBC system, especially at low correlation. Therefore, we can employ the precoding technique in transmit node to improve the performance of relay system.

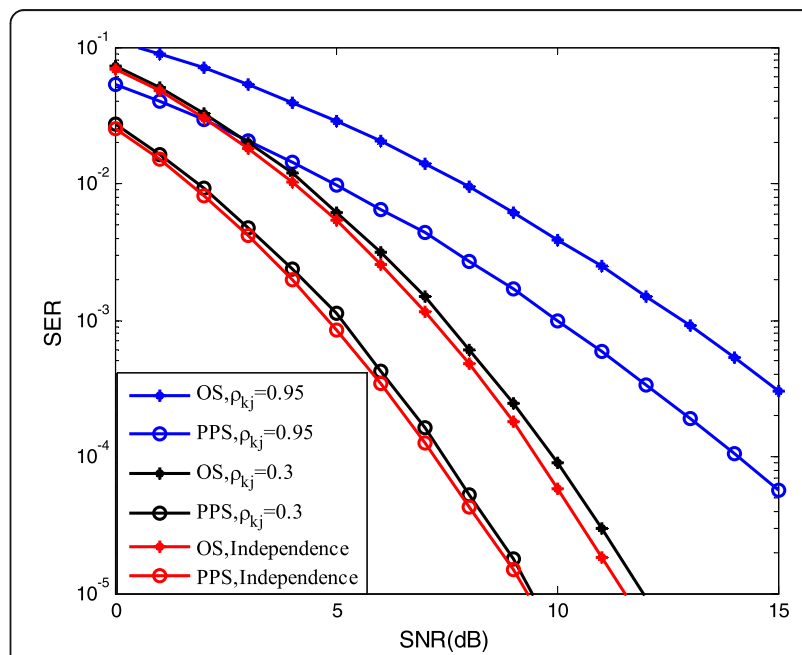

Fig. 5 Comparison between the performance of the precoding-plusSTBC and only STBC relay system based on LLR 


\subsection{Scenario 2}

The following four figures depict the SER performance of relay system with imperfect channels.

Figure 6 illustrates the SER comparisons of various schemes and spatial correlation coefficients between antennas for different number of relays $(I=2,3)$ and correlation fading $\left(\rho_{k j}=0.3,0.95\right)$. It is evident that a better SER performance is provided by the scheme LLR-based. Moreover, when the correlation is fixed, the performance gap between the schemes based on LLR and SNR increases as the number of relays increases. And when the number of relays is fixed, the gap increases as the correlation becomes weak. This is consistent with the performance under perfect CSI.

Results of Fig. 7 provide the comparisons of precoding-plus-STBC system and only precoding system adopt LLR criterion when $\rho_{k j}=0.3,0.8,0.95$, and $I=2$. It is observed from Fig. 7 that the precoding-plus-STBC method provides much better SER performance than only precoding methods. This is because the STBC technology provides considerable performance gain. For example, at a SER of $10^{-3}$, the precoding-plus-STBC obtain a gain of approx. $1.5 \mathrm{~dB}, 2.5 \mathrm{~dB}$, and $4 \mathrm{~dB}$ when $\rho_{k j}=0.3,0.8$ and 0.95 respectively. We can see that the performance gain increases with the increase of correlation, which agrees also with the results in perfect channel.

Finally, we investigate the SER of the proposed scheme based on LLR versus the transmit and receive correlations in two kinds of system, precoding-plus-STBC system and only STBC system. Figure 8 demonstrates that the SER versus correlation coefficient at the transmitter when correlations at the receiver is fixed $\rho_{k j}=0.9$ and $\mathrm{SNR}=10 \mathrm{~dB}$. On the one hand, the performance gap between the precoding-plus-STBC system and only STBC system increases as the transmit correlation increases

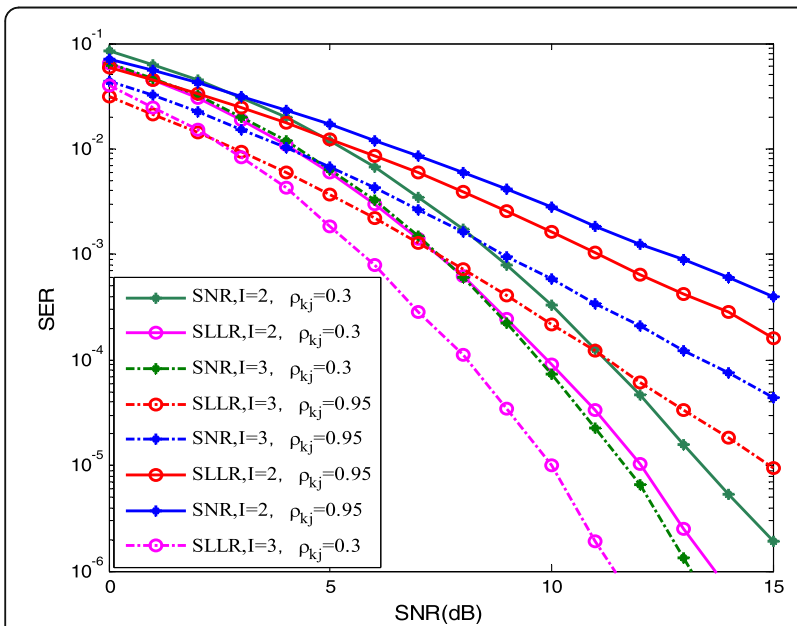

Fig. 6 The performance comparison with different number of relays and correlation fading

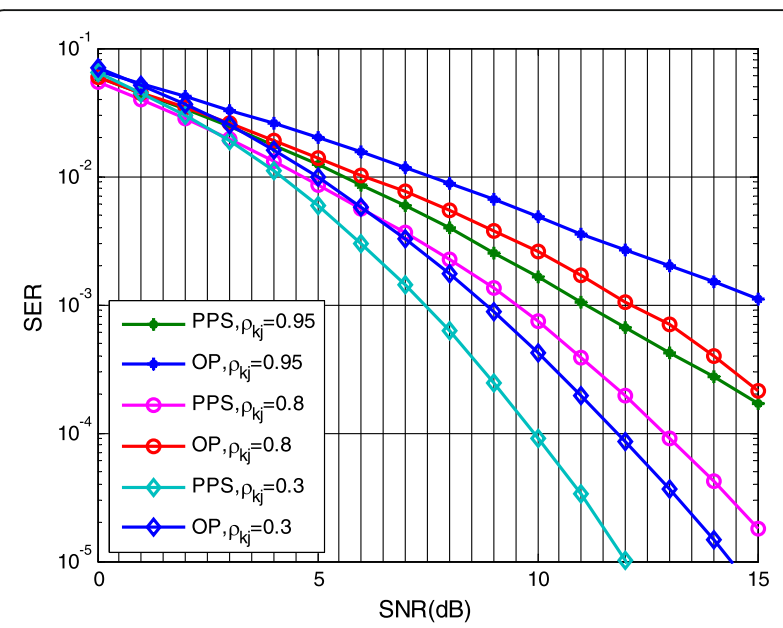

Fig. 7 Comparison between the performance of the precoding-plusSTBC and only precoding relay system with imperfect CSI

and disappears when the correlation is low. On the other hand, with the increase of the number of relays, the improvement is also increasing. Figure 9 displays the SER versus correlation coefficient at the receiver when correlation at the transmitter is fixed $\rho_{k j}=0.3,0.9$ and $\mathrm{SNR}=10 \mathrm{~dB}$. If the transmit correlation is small $\left(\rho_{k j}=0.3\right)$, both precoding-plus-STBC system and only STBC system show similar performance for all values of the receive correlation. Only when the transmit correlation is large $\left(\rho_{k j}=0.9\right)$, the superiority of the proposed method is obvious. Moreover, the improvement decreases with the enhancement of correlation. This observation agrees with a well-known result that the power allocation of the precoding converts into the classical equal power allocation in the case of receive correlation only.

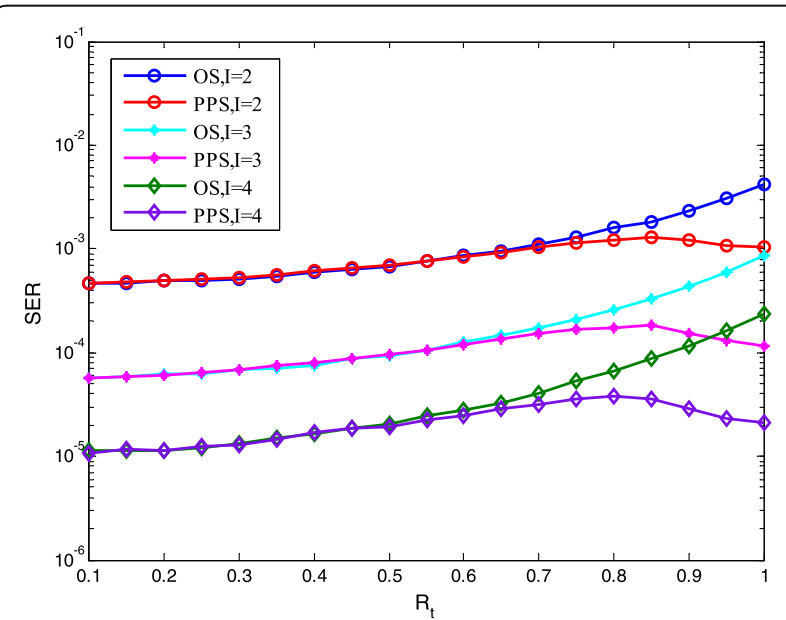

Fig. 8 SER performance versus the transmit correlation with constant receive correlation 


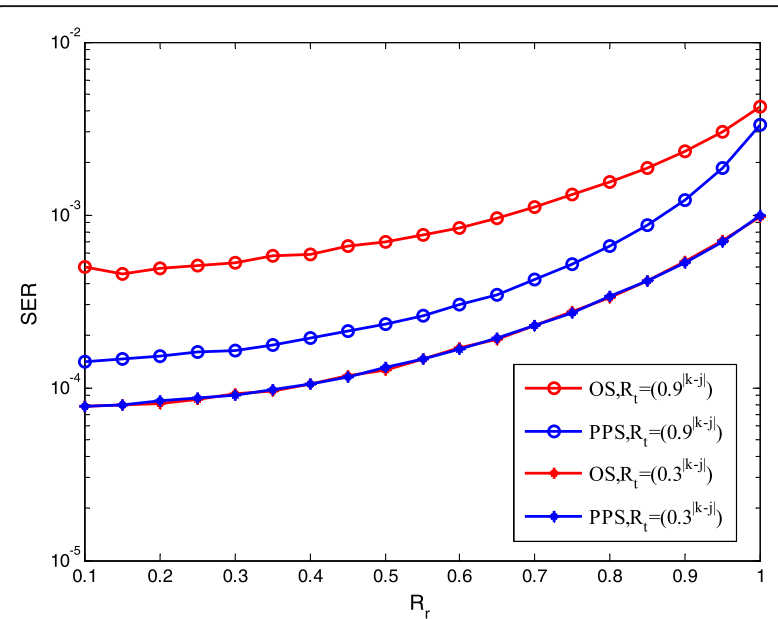

Fig. 9 SER performance versus the receive correlation with constant transmit correlation

\section{Conclusions}

In this paper, we investigate the SER performance of a relay selection LLR-based system with precoding-plusSTBC employed in the source and relay nodes. Compared with the traditional relay selection SNR-based, the relay selection scheme chooses the best relay based on LLR and obtains better performance with prefect CSI and imperfect CSI. Moreover, the average SER performance of proposed scheme is analyzed. Simulation results show that a better performance can be obtained with the higher number of relay nodes and the smaller correlation fading coefficient. Moreover, the performance of precoding combined with STBC system is superior to that of the only precoding system and the degree of improvement increases with the enhancement of the correlation. Meanwhile, the performance of the precodingplus-STBC system is also better than that of the only STBC system, but the gap decreases with the enhancement of the correlation.

\begin{abstract}
Abbreviations
AF: Amplify-and-forward; AWGN: Additive white Gaussian noise; BPSK: Binary phase-shift keying; CSI: Channel state information; DF: Decode-and-forward; LLR: Log-likelihood ratio; MIMO: Multiple-input multiple-output; MISO: Multipleinput single-output; MRC: Maximum ratio combining; MSE: Mean square error; OP: Only precoding; OS: Only STBC; PPS: Precoding-plus-STBC; SC: Successivecancelation; SER: Symbol error rate; SNR: Signal-to-noise ratio; STBC: Space-time block codes
\end{abstract}

\section{Acknowledgements}

The authors would gratefully acknowledge the grants from the National Natural Science Foundation of China (61571250), the Natural Science Foundation of Ningbo City of China (2015A610121), and the K.C. Wong Magna Fund of Ningbo University.

Availability of data and materials

The authors declare that all the data and materials in this manuscript are available.

\section{Authors' contributions}

YZ conceived and designed the study and then performed the simulation experiments and wrote the paper. YL reviewed and edited the manuscript. Both authors read and approved the final manuscript.

\section{Competing interests}

The authors declare that they have no competing interests.

\section{Publisher's Note}

Springer Nature remains neutral with regard to jurisdictional claims in published maps and institutional affiliations.

\section{Author details}

${ }^{1}$ College of Information Science and Engineering, Ningbo University, NingBo 315211, Zhejiang, China. ${ }^{2}$ College of Mathematics, Physics and Information Engineering, Zhejiang Normal University, Jinhua 321004, Zhejiang, China.

Received: 12 January 2018 Accepted: 11 October 2018

Published online: 22 October 2018

\section{References}

1. S. Baghel, L.S. Pillutla, Relay based wireless networks with joint modulation, power allocation and relay selection, 2017 IEEE International Conference on Advanced Networks and Telecommunications Systems (ANTS) (2017), pp. 1-6

2. S. Dang, G. Chen, J.P. Coon, Outage performance of two-hop OFDM with index modulation and multi-carrier relay selections, IEEE Wireless Communications Letters (2018), pp. 1-4

3. E. Li, X. Wang, Z. Wu, G. Yang, Outage performance of DF relay selection schemes with outdated CSI over Rayleigh fading channels. IET Commun. 12(8), 984-993 (2018)

4. S. Silva, M. Ardakani, C. Tellambura, Relay selection for cognitive massive MIMO two-way relay networks, 2017 IEEE Wireless Communications and Networking Conference (WCNC) (2017), pp. 1-6

5. P. Xu, Z. Yang, Z. Ding, Z. Zhang, Optimal relay selection schemes for cooperative NOMA. IEEE Trans Veh. Technol. 67(7), 7851-7855 (2018)

6. Y. Zhang, J. Ge, Joint antenna-and-relay selection in MIMO decode-andforward relaying networks over Nakagami-m fading channels. IEEE Signal Processing Letters 24(4), 456-460 (2017)

7. T. Tao, A. Czylwik, Beamforming design and relay selection for multiple MIMO AF relay systems with limited feedback, IEEE 77th Vehicular Technology Conference (VTC Spring) (2013), pp. 1-5

8. Y. Su, L. Jiang, C. He, Q. Xi, Relay selection and beamforming with outdated CSI for AF MIMO relay systems, 2015 IEEE International Conference on Communication Workshop (ICCW) (2015), pp. 544-548

9. L. Samara, A. Gouissem, R. Hamila, Hybrid full-duplex and alternate multiple relay selection and beamforming in AF cooperative networks, vol 6 (2018), pp. 28467-28477

10. H. Moharrer, A. Olfat, Joint relay selection and cooperative beamforming in two-hop multi-relay decode-and-forward networks. IET Commun. 8(18), 3245-3253 (2014)

11. A. Sheikh, A. Olfat, New beamforming and relay selection for two-way decode-and-forward relay networks. IEEE Transactionson Vehicular Technology 65(3), 1354-1366 (2016)

12. H. Yajie, Y. Cheng, W. Yang, Performance analysis of a cooperative SC-FDE D-STBC transmission system with best relay selection, 2015 5th International Conference on Information Science and Technology (ICIST) (2015), pp. 475-781

13. W. Jiang, T. Kaiser, A.J.H. Vinck, A robust opportunistic relaying strategy for co-operative wireless communications. IEEE Trans. Wirel. Commun. 15(4), 2642-2655 (2016)

14. V.B. Olivatto, R.R. Lopes, E.R. de Lima, Simplified method for log-likelihood ratio approximation in high-order modulations based on the Voronoi decomposition. IEEE Trans. Broadcast. 63(3), 583-589 (2017)

15. C. Condo, F. Ercan, W.J. Gross, Improved successive cancellation flip decoding of polar codes based on error distribution, 2018 IEEE Wireless Communications and Networking Conference Workshops (WCNCW) (2018), pp. 19-24

16. Y.G. Kim, S.W. Kim, Optimum selection diversity for BPSK signals in Rayleigh fading channels. IEEE Trans. Commun. 49(10), 1715-1718 (2001)

17. Y.G. Kim, S.W. Kim, Optimum selection combining for M-ary signals in frequency-nonselective fading channels. IEEE Transnsactions Communications 53(1), 84-93 (2005) 
18. Y.G. Kim, N.C. Beaulieu, SEP of decode-and-forward cooperative systems with relay selection in Nakagami-m fading channels. IEEE Transactionson Vehicular Technology 64(5), 1882-1894 (2015)

19. H. Nourollahi, R.S. Kandovan, Log-likelihood ratio-based relay selection algorithm in two-way relay channel with physical network coding, 2017 IEEE 13th Malaysia International Conference on Communications (MICC) (2017) pp. $74-78$

20. A. El-Mahdy, A. Waleed, Log-likelihood ratio-based relay selection algorithm for cooperative communications, 2015 International Conference on Communications, Signal Processing, and their Applications (ICCSPA) (2015), pp. 1-6

21. A. Hjørungnes, D. Gesbert, Precoding of orthogonal space-time block codes in arbitrarily correlated MIMO channels: iterative and closed-form solutions. IEEE Trans. Wirel. Commun. 6(3), 1072-1082 (2007)

22. B.K. Chalise, Y.D. Zhang, M.G. Amin, Precoder design for OSTBC based AF MIMO relay system with channel uncertainty. IEEE Signal Processing Letters 19(8), 515-518 (2012)

23. Y. Zhang, Y. Li, G. Wang, M. Jin, Precoding of space-time block codes for relay networks over correlated MIMO channels. Wirel. Pers. Commun. 84(2), 1387-1400 (2015)

24. E. Biglieri, R. Calderbank, A. Constantinides, et al., MIMO Wireless Communications (Cambridge University Press, Cambridge, 2007), pp. 62-98

25. A. Paulraj, R. Nabar, D. Gore, Introduction to Space-Time Wireless Communications (Cambridge University Press, Cambridge, 2003)

26. C. Oestges, B. Clerckx, MIMO Wireless Communications-From Real-World Propagation to Space-Time Code Design (Academic Press of Elsevier, London, 2010)

27. Z. Youyan, Z. Changjiang, Performance of MIMO system with transmit antenna selection based on LLR, 2009 IEEE international conference on communications technology and applications (2009), pp. 550-554

28. L. Yang, Q.T. Zhang, Performance analysis of MIMO relay wireless networks with orthogonal STBC. IEEE Transactionson Vehicular Technology 59(7), 3668-3674 (2010)

\section{Submit your manuscript to a SpringerOpen ${ }^{\circ}$ journal and benefit from:}

- Convenient online submission

- Rigorous peer review

- Open access: articles freely available online

High visibility within the field

- Retaining the copyright to your article

Submit your next manuscript at $\boldsymbol{\nabla}$ springeropen.com 